\title{
Finite Element Analysis of Uncemented Total Hip Replacement: the Effect of Bone-Implant Interface
}

\author{
Nur Faiqa Ismail ${ }^{1 *}$, Solehuddin Shuib ${ }^{2}$, Muhd Azman Yahaya ${ }^{3}$, Ahmad Zafir Romli ${ }^{4}$, Amran Ahmed Shokri ${ }^{5}$ \\ ${ }^{* 1}$ Faculty of Mechanical Engineering, Universiti Teknologi MARA,40450 Shah Alam, Selangor \\ ${ }^{2}$ Institute of Science, Universiti Teknologi MARA, 40450 Shah Alam, Selangor \\ ${ }^{3}$ School of Science Health, Universiti Sains Malaysia, 16150 Kubang Kerian, Kelantan \\ *Corresponding author E-mail: solehuddin2455@salam.uitm.edu.my
}

\begin{abstract}
Most uncemented total hip replacements (THR) rely on press-fit for the initial stability and thus lead to the secondary fixation which is biological fixation. Choosing the accurate interference fit may have a great effect on implant stability and implant loosening prevention. Implant loosening is the most reported problem where it leads the increasing of micromotion at the bone-implant interface due to insufficient primary fixation. By having sufficient stability or fixation after surgery, minimal relative motion between the prosthesis and bone interfaces allows osseointegration to occur. Therefore, it will provide a strong prosthesis-to-bone biological attachment. The aim of this study was to evaluate the effect of bone-implant interface for uncemented hip implant. In this study, a three-dimensional model of hip implant was designed and analysed by using commercial Finite Element Software namely, ANSYS WORKBENCH V15 software in order to investigate the bone-implant interface effect using the chosen implant design. The value of interference fit $(\delta=0.01,0.05,0.10$ and $0.50 \mathrm{~mm})$ and coefficient of friction $(\delta=0.15,0.40$ and 1.00$)$ were used to simulate the bone-implant interface. It was found that the interference fit of $0.50 \mathrm{~mm}$ was sufficient to achieve the primary fixation and also the best fitting; thus, the implant loosening can be minimized. The interference fit of $0.50 \mathrm{~mm}$ was the minimal value to achieve fixation, while the coefficient of friction did not affect the bone-implant interface.
\end{abstract}

Keywords: Uncemented total hip replacement, finite element analysis, micromotion, interference fit, coefficient of friction.

\section{Introduction}

Total hip replacement has played an important role and continued to be a great remedy to overcome problems related to the human hip. This surgery involve replacing the damaged upper end of thighbone or femur and hip socket in the pelvic bone with prosthetic implants. Generally THR is done to reduce severe joint damage and to relieve pain caused commonly by arthritis. Besides, it also helps to improve the human hip function. Many factors that leads to this replacement such as osteoarthritis, rheumatoid arthritis, avascular necrosis or even childhood hip disease. All of these diseases make the human hard to move their hip freely; thus, human daily activities may interrupted and limited.

Furthermore, the elderly bone normally weaker than the younger. As the proportion of elderly people increases in many countries, the economic and social consequences of aging are also increases. An elderly often associated with a disease which recognized as osteoporosis and consequently becoming a significant public health burden. Osteoporosis occurred when the human bone had tissue loss and becomes brittle and fragile due to either insufficient of calcium or vitamin D and consequently increase the risk of bone fracture.

In general, total hip replacement (THR) comes out into two types; cemented and uncemented total hip replacement. Each of replacement types have their own advantages and disadvantages where several factors need to be considered during the selection. Normally the surgeon will give the suggestion to their patients regarding to their age, lifestyle, occupation and also their bones' quality.

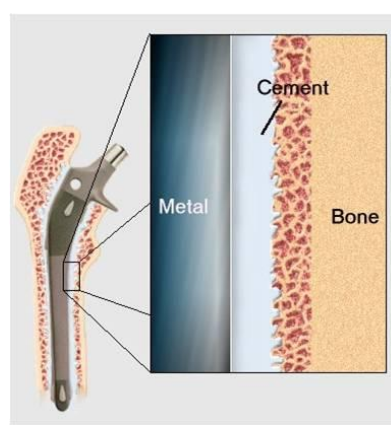

(a)

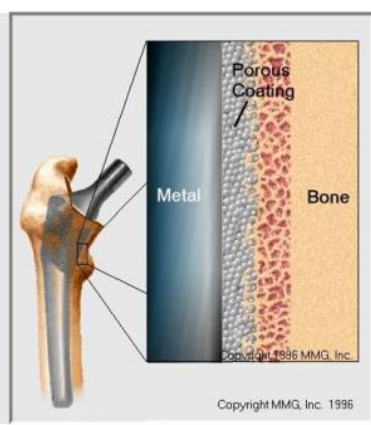

(b)
Fig. 1: (a) Cemented total hip replacement (b) Uncemented total hip replacement [1]

The first replacement that being introduced was cemented total hip replacement. This replacement normally use PMMA cement type to hold the implant inside the metaphyseal bone canal. Eventhough this replacement has been used for a long period, however, there are also many reported cases which required a revision. Some drawback of cemented implant is breaking off cemented particles. The implant become loosening due to this cement breakdown. Moreover, the cement debris can also irritate the surrounding tissue and cause inflammation and it even can be life-threatening where the debris can get into the bloodstream and end up in the human lungs. 
Hence, uncemented hip implants were designed and introduced later conjunction to eliminate the issues associated with the cemented hip implants [2]. Uncemented hip replacement is generally a press-fitting process of a hip prosthesis into the femoral bone where it use biological fixation to hold the implant inside of the bone. This replacement is succeeds once the primary stability is achieved and consequently leads to the secondary fixation via osseointegration or also known as bony ingrowth surrounding the prosthesis.

The main key of a successful cementless THR is by controlling the bone-implant interface. Nevertheless, achieving good primary fixation is one crucial importance in this replacement to ensure good short-term and long-term outcomes [3]. Lack of primary fixation could result into unpleasant conditions in terms of clinical and also mechanical which are thigh pain, loosening of prosthesis and micromotion [4].

Typically total hip replacement was introduced for a long period usage, however, there are still cases occurred that leads to revision. The THR revision is done because of the artificial implant did not achieve its intended standards or also called as THR failure. The Australian Orthopaedic Association National Joint Replacement Registry THR data highlighted the most common cases that require THR revision are loosening, prosthesis dislocation, fracture, and infection as well. Fig. 2 shows the summary of THR revision data in Australia as by 2017. From the data in Fig. 2, it is apparent that the highest causes of revision was implant loosening. The main factor of the implant loosening was the insufficient of implant stability.

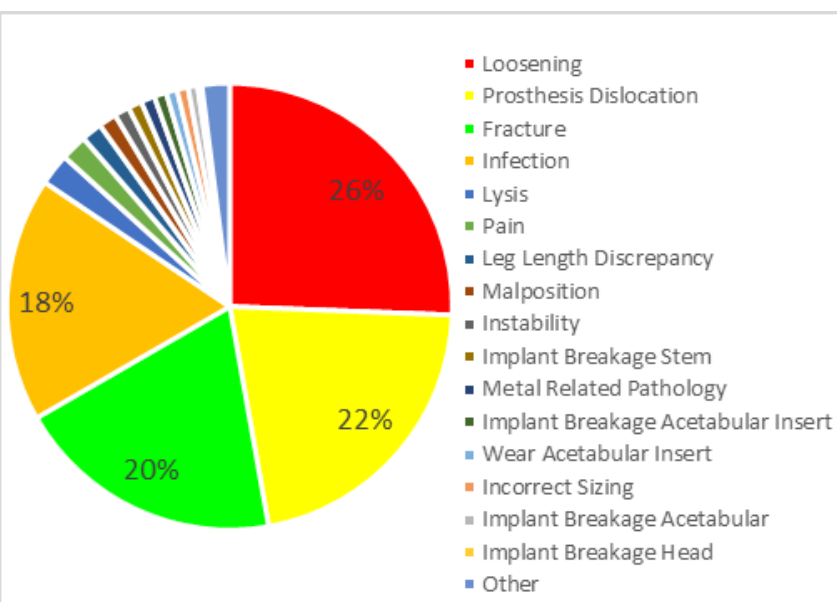

Fig. 2: The most reported cases that required revision in Australia by 2015 [5]

It is necessary to measure the relative motion between the boneimplant interfaces in order to maintain the stability of the hip prosthesis under various human physiological loads. The relative motion between the bone-implant interfaces could give different result where it can be fit, loose or too tight depending on the amount being set at the primary stage of the surgery. Meanwhile, other researcher argued that introducing shorter stem design could maximise the stability of the implant [2].

Large relative motion will reduce the chances of osseointegration which eventually leads to the implant loosening and failure of the replacement. Micromotion may be limited by providing an adequate press-fit between the bone-implant interfaces at the time of implantation of the prosthesis [6]. The press-fit fixation is an immediate post-operative which is implemented by a mechanical correlation between the frictional at the bone-implant interface and the bone stresses resulting from the implantation process [7]. For the long term fixation, a complex process of biological will enhance the bony ingrowth or osseointegration $[8,9]$ which provides the secondary fixation.

The unstable prosthesis may cause obstacle such as thigh pain [10-12] and the prosthesis will eventually loosen [12, 13]. The bony ingrowth is hindered by an excessive micromotion at the bone-implant interface where this excessive micromotion prevents calcification of the tissue within the pores, and as a result, only fibrous tissue fixation occurs. In addition, the bony ingrowth might not occur if the relative motion between bone-implant is high. In order to achieve greater long-term implant stability through the ingrowth of the bone, minimal micromotion is required. Thus, it is important to determine the limiting micromotion value that inhibits stable bony ingrowth. The objective of this study is to evaluate the effect of the bone-implant interfaces at different interference fit and coefficient of frictions.

\section{Materials and Method}

In general, interference fit is a fastening between two parts where it provide a connection between them as the external dimension must slightly exceed the internal dimension of another part to form a press-fit. This concept often been used for hub and shaft assembly. This connection will form the forces of friction between the mating surfaces as it enable load transmission. This is because the shaft has larger diameter than the hub in order to engage between these two parts. Interference fit has been widely used for medical application including for dental implants and hip implants as well. Fig. 3 shows two different bonded materials which indicated cortical bone and implant where equal axial force, $\mathrm{F}(\mathrm{N})$ were exerted on them.

Press-fit force calculation: By using the Hooke's Law and the fact that the stress was force/ area; then,

$\frac{\sigma}{E}=\frac{\sigma_{1}}{E_{1}}+\frac{\sigma_{2}}{E_{2}}$

$\frac{F}{A E}=\frac{F_{1}}{A_{1} E_{1}}+\frac{F_{2}}{A_{2} E_{2}}$

By knowing that the deformation on parallel material illustrate the Voight's model; thus,

$E=\frac{1}{A}+A_{1} E_{1}+A_{2} E_{2}$

Substitute the equation 3 into the equation2,

$F_{b}=\frac{A_{b} E_{b} F}{A_{i} E_{\tilde{i}}+A_{b} E_{b}}$

$F_{\text {iิ }}=\frac{A_{\tilde{i}} E_{\text {i⿱ }} F}{A_{\tilde{i}} E_{\tilde{i}}+A_{b} E_{b}}$

Where:

$\mathrm{F}_{\mathrm{b}}=$ Force for bone

$F_{i}=$ Force for implant

$\mathrm{A}_{\mathrm{b}}=$ Area for cortical bone

$\mathrm{A}_{\mathrm{i}}=$ Area for implant

$\mathrm{E}_{\mathrm{b}}=$ Young Modulus for bone

$\mathrm{E}_{\mathrm{i}}=$ Young Modulus for implant

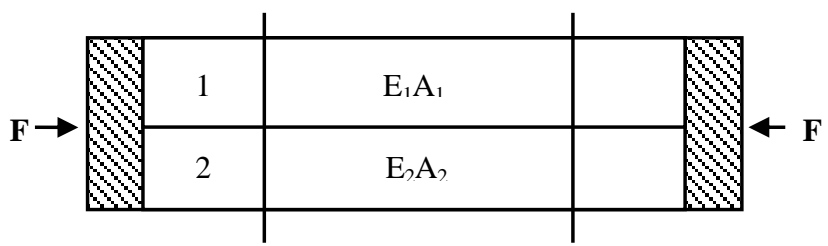

Fig. 3: An equal force were subjected on two different bonded materials 
A solid cylindrical model for stem section was constructed by using ANSYS Workbench Software; comprised of 420 element with length of $60 \mathrm{~mm}$ and an adjustable outer diameter in order to create variable degrees of press-fit around of the femur. The type of material used for implant is one of the main components for successful surgery where it influences the stability of the implant; hence resulting long term fixation to the patients. Thus, Titanium Ti-6Al-4V was chosen because it is viewed as the most biocompatible metal due to the imperviousness to corrosion from natural liquids, high fatigue limit, limit for osseointegration and bioidleness, where it is registered as biomaterials in ASTM standard [10].

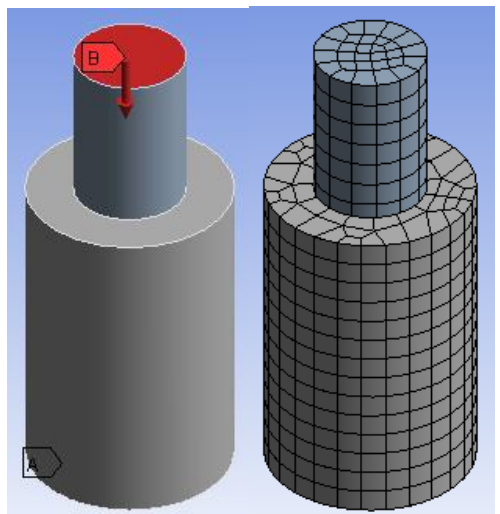

Fig. 4: Loading and boundary condition and meshing of the bone and implant

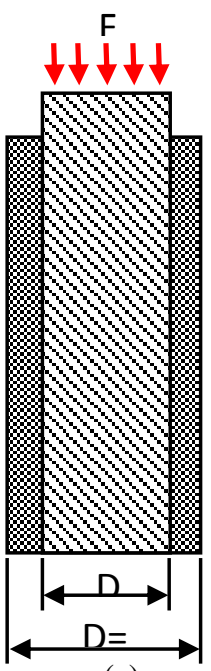

(a)

Diameter, $\mathrm{D}$ was defined as an adjustable outer diameter to create range of interference fit; $\delta=0.01,0.05,0.10$ and $0.50 \mathrm{~mm}$

From top view,

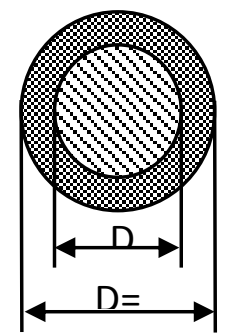

(b)

Fig. 5: (a) Diagram of the model showing the dimensions of cortical bone and the implant and the load direction (b) Diagram of the model from the top view

Next, for the cortical bone model, a hollow-cylindrical was constructed which comprised of 424 element, with length of $40 \mathrm{~mm}$, outer diameter of $26 \mathrm{~mm}$ and inner diameter of $14 \mathrm{~mm}$. This femo- ral bone was assigned as homogenous, isotropic and linearly elastic as well. This bone was set as fixed at the distal end and the loading condition is illustrated in Fig. 4. The dimension for the bone and implant model from front view as in the Fig. 5(a) and from top view as in the Fig. 5(b).

Meanwhile, the properties to be used in finite element analysis for all the materials consists of different Young modulus, E (MPa) and poisson ratio which shown in Table 1.

Table 1: Material properties of the implant and the cortical bone [14]

\begin{tabular}{|l|l|l|}
\hline Material & $\begin{array}{l}\text { Young Modulus, E } \\
(\mathrm{MPa})\end{array}$ & Poisson ratio \\
\hline Titanium & 100,000 & 0.3 \\
\hline Cortical Bone & 14,200 & 0.3 \\
\hline
\end{tabular}

In order to simulate the bone-implant interface, four different interference values were selected in which each of them was evaluated along with three different coefficients of friction. The selected values for the interference fit were $0.01,0.05,0.10$ and 0.50 $\mathrm{mm}$ corresponding to the adjustable stem outer diameter of 14.01, $14.05,14.10$ and $14.50 \mathrm{~mm}$ respectively. Each selected values of the interference fit showed different interference effect where the interference value of $0.01 \mathrm{~mm}$ correlated to a "line-to-line" fit. For reflecting a "slight" press-fit, interferences of 0.05 and $0.10 \mathrm{~mm}$ were chosen while $\delta=0.50 \mathrm{~mm}$ was chosen to correspond the present clinical approach.

In this study, three different values of coefficient of friction were selected. They were $\mu=0.15, \mu=0.40$ and $\mu=1.0$ respectively. Each of the coefficient of friction value represents different condition; $\mu=0.15$ indicates a well-lubricated at the bone-implant interface while $\mu=0.40$ indicates a poor lubrication between the bone and implant. Next, it is said that the suitable coefficient of friction between bead porous-coated titanium plate and cortical bone was at the range from 0.45 to 1.3 . Thus, for the parametric study purpose, $\mu=1.0$ was chosen as a practical upper bound.

The analysis consisted of two (2) steps: (1) creating press-fit between the bone-implant interface using the selected values of interference fit and coefficient of friction and (2) the application of static load.

Loss of fixation, for the given $\delta$ and $\mu$, describes the minimum axial load that leads to the global slipping between the bone and implant. This represents that the entire interface nodes are slipping at the same time; hence this load is called as push-out load. This work will be validated with Shultz et al. (2006) works where the parameters was taken for benchmarking; interference fit and coefficient of friction values.

\section{Results and Discussion}

This study demonstrated the consequence of the interference fit as it included only a small level of interference which changed the stability of the implant inside the cortical bone. Based on the result obtained in this present study, the visco-elastic behaviour of the human cortical bone has a diminishing effect upon the push-out strength for distally press-fit femoral implants within a short period of time immediately followed by the implantation process.

If the diameter of the inner bone was $14.00 \mathrm{~mm}$ and the implant was $14.01 \mathrm{~mm}$, the bone-implant interface would be $\delta: 0.01 \mathrm{~mm}$. The effect of the von Mises stress at this interface can be seen in Fig.6.

As can be seen in Fig. 6, the von Mises Stress increases as the load applied increases. This shows that this amount of interference fit is insufficient enough to create the press-fit between the bone and the implant where it could be too loose to hold the implant. Hence, the implant might be lost under physiological loading.

Fig. 7 shows the von Mises stress at the interference fit of $0.05 \mathrm{~mm}$. When the diameter of the implant was increased to $14.05 \mathrm{~mm}$, the von Mises stress became stagnant or plateau as the load applied increased. This behaviour demonstrated that this 
interference fit is the minimal interference fit which has been suggested by Kadir et al. where they stated that the optimal level of

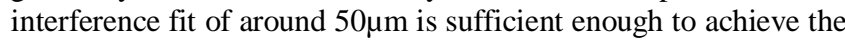
primary fixation [3]. They also stated that the value of micromotion between the ranges of 50 to $150 \mu \mathrm{m}$ will disrupt the osseointegration process; thus, the primary fixation will not achieve and consequently leads to the implant failure. Besides that, higher interference fit will cause excessive stresses which can lead to periprothetic fracture during implantation [15-16].

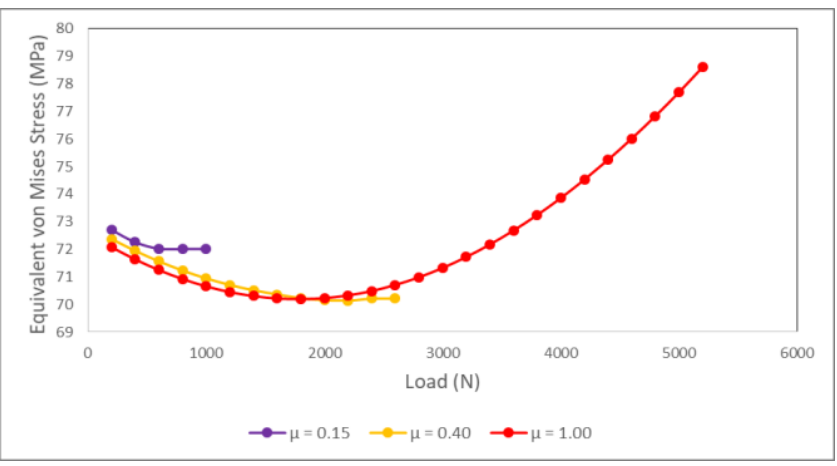

Fig. 6: Equivalent von Mises Stress versus load for different coefficient of friction at the interference of $0.01 \mathrm{~mm}$

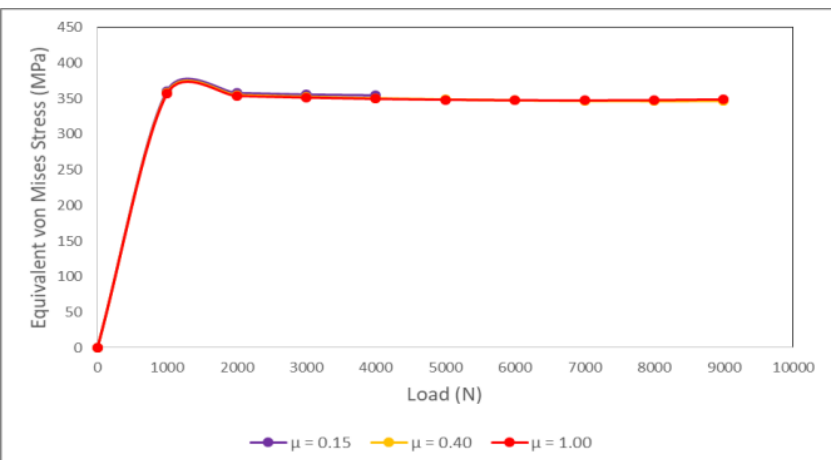

Fig. 7: Equivalent von Mises Stress versus load for different coefficient of friction at the interference of $0.05 \mathrm{~mm}$

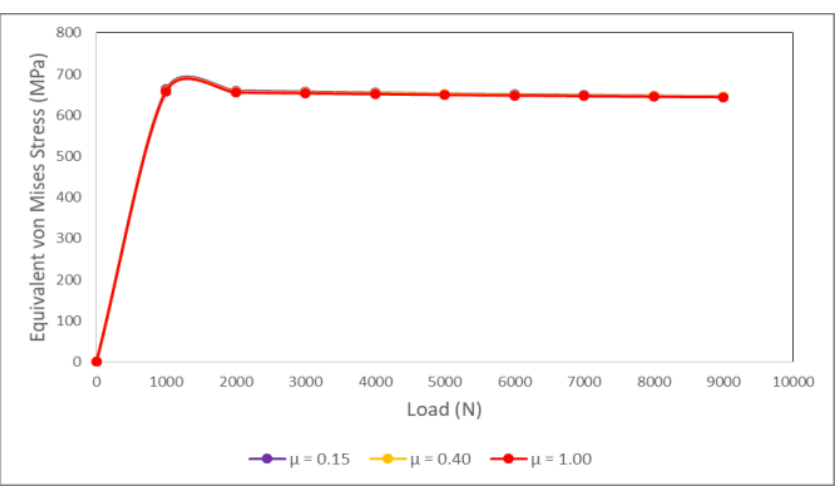

Fig. 8: Equivalent von Mises Stress versus load for different coefficient of friction at the interference of $0.10 \mathrm{~mm}$

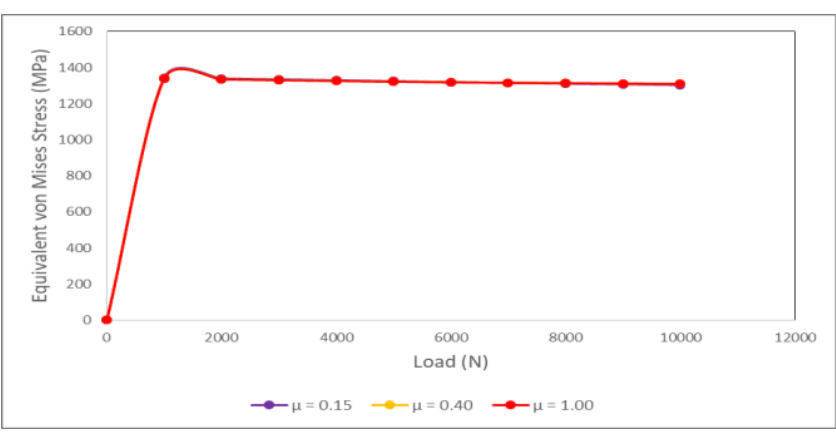

Fig. 9: Equivalent von Mises Stress versus load for different coefficient of friction at the interference of $0.20 \mathrm{~mm}$
In addition, we found that there is no increment of fixation strength beyond certain interference fit value. This is possibly due to the excessive stresses formation during the insertion which causes bone damage; thus, limiting the additional stability of the implant [17]. Moreover, Shultz et al. (2006) considered that interference fit of $100 \mu \mathrm{m}$ can diminish between the bone and implant and they reported this level of interference as a threshold value [18]. It can be seen that the von Mises Stress at $\delta=0.10 \mathrm{~mm}$ is higher compared to $\delta=0.05 \mathrm{~mm}$ as shown in Fig. 7 .

However, Shultz et al. (2006) again claimed that hoop stresses in the bone would elicit visco-elastic bone relaxation by approximately $50 \%$ at the interference fit of $100 \mu \mathrm{m}$. In other words, if the interference fit of $100 \mu \mathrm{m}$ was chosen, the bone would relax and represent an effective interference fit of $50 \mu \mathrm{m}$. Thus, there must be an effective interference fit after the bone relaxation of at least $25 \mu \mathrm{m}$ during the implantation. However, the coefficient of friction does not necessarily translate to a greater stability where the final position of the prosthesis may be affected under a given implantation force.

Interference fit is a very important parameter in order to achieve the best fitting of many implants. This is because the changing of the implant size or bone cavity is much easier to control.

Previously, many studies reported the effect of interference fit on the mechanical performance of the orthopedic implants or dental press-fit $[2,3,9,19-20]$. In general, the trend illustrates the fixation improvement by increasing the interference fit; however, it also may lead to bone damage.

A successful uncemented total hip replacement (THR) depends on the initial stability at the bone-implant interface. Stability is achieved through press-fit process of a femoral implant compatible with the endosteal geometry of the bone. Press-fitting may increase the intraoperative proximal femoral fractures for the uncemented THR at the rates of $2.95-27.8 \%$ [21] when there is insufficient implant stability. Hence, defining the sensible range of interference fit is very important and critical in order to achieve the best fitting and consequently preventing bone or implant failure.

\section{Conclusion}

The uncemented THR is successful once the primary fixation is achieved which consequently leads to the secondary fixation through a biological process namely osseointegration. The secondary fixation will not occur when there is insufficient amount of interference fit. Four different interference fits with three different coefficients of friction were analyzed for the bone-implant interface effect. The results obtained confirmed that the fixation strength is influenced by the amount of interference fit to a large extent. Thus, considering higher interference fit may improve the implant fixation and it depends on the implant morphological surface. This study supports that the implant can achieve the best fitting and stability inside the proximal femur metaphyseal region with minimal interference fit of $50 \mu \mathrm{m}$; therefore, the implant or bone failure can be prevented.

\section{Acknowledgement}

The authors would like to thank the Research Management Institute (RMI) of Universiti Teknologi MARA (UiTM) and the Ministry of Education, Malaysia for financial support and facilitating this project through Research GIP GRANT awards 600IRMI/MyRA 5/3 (036/2017). The authors also would like to express deepest appreciation to Assoc. Prof. Ir. Dr. Jamaluddin Mahmud, whose contribution in stimulating suggestions and encouragement throughout this project. 


\section{References}

[1] Morphopedics: Where technology and orthopedics collides. Retrieved from http://morphopedics.wikidot.com/total-hiparthroplasty

[2] M. T. Bah et al., "Inter-subject variability effects on the primary stability of a short cementless femoral stem," J. Biomech., vol. 48, no. 6, pp. 1032-1042, 2015.

[3] M. R. Abdul-Kadir, U. Hansen, R. Klabunde, D. Lucas, and A Amis, "Finite element modelling of primary hip stem stability: The effect of interference fit," J. Biomech., vol. 41, no. 3, pp. 587-594, 2008 .

[4] M. Nizam Ahmad, S. Solehuddin, A. Y. Hassan, A. S. Amran, M .I .Z. Ridzwan and M. N. Mohd. Ibrahim, "Application of Multi Criteria Optimization Method in Implant Design to Reduce Stress Shielding," Journal of Applied Sciences, 7, 2007.

[5] AOA, "Australian Orthopaedic Association National Joint Replacement Registry," in Annual Report, 2017.

[6] M. I. Z. Ridzwan, S. Solehuddin \& A.Y, Hassan \& A. S. Amran. "Effects of Increasing Load Transferred in Femur to the BoneImplant Interface”, Journal of Applied Sciences, 6, 2006.

[7] S. Berahmani et al., "An experimental study to investigate biomechanical aspects of the initial stability of press-fit implants," $J$. Mech. Behav. Biomed. Mater. vol. 42, pp. 177-185, 2015.

[8] H. S. Alghamdi, J. J. J. P. van den Beucken, and J. A. Jansen, "Osteoporotic Rat Models for Evaluation of Osseointegration of Bone Implants," Tissue Eng. Part C Methods, vol. 20, no. 6, pp. 493-505, 2014.

[9] M. R. Abdul Kadir and N. Kamsah, "Interface micromotion of cementless hip stems in simulated hip arthroplasty," Am. J. Appl. Sci., vol. 6, no. 9, pp. 1682-1689, 2009.

[10] K. Colic, A. Sedmak, A. Grbovic, U. Tatic, S. Sedmak, and B. Djordjevic, "Finite element modeling of hip implant static loading," Procedia Eng., vol. 149, no. June, pp. 257-262, 2016.

[11] R. Zdero, Z. S. Bagheri, M. Rezaey, and E. H. Schemitsch, "The Biomechanical Effect of Loading Speed on Metal-on-UHMWPE Contact Mechanics," pp. 28-34, 2014.

[12] N. Arden and M. C. Nevitt, "Osteoarthritis: Epidemiology," Best Pract. Res. Clin. Rheumatol., vol. 20, no. 1, pp. 3-25, 2006.

[13] M. Rafiq, A. Kadir, and U. N. Hansen, "The Effect of Physiologica Load Configuration on Interface Micromotion in Cementless Femoral Stems," Tissue Eng., no. 23, pp. 50-61, 2007.

[14] Shuib, S., Sahari, B. B., Voon, W. S. \& Arumugam, M. 2012 In: Trends in Biomaterials and Artificial Organs. 26, 2, p. $103-1064 \mathrm{p}$

[15] G. D. Dumont, J. R. Zide, and M. H. Huo, "Periprosthetic Femur Fractures: Current Concepts and Management," Semin. Arthroplasty, vol. 21, no. 1, pp. 9-13, 2010.

[16] R. Nowak, M., Kusz, D., Wojciechowski, P., Wilk, "Risk factors for intraoperative periprosthetic femoral fractures during the total hip arthroplasty," Polish Orthop. Traumatol., no. 77, pp. 59-64, 2012.

[17] S. Berahmani, D. Janssen, and N. Verdonschot, "Experimental and computational analysis of micromotions of an uncemented femoral knee implant using elastic and plastic bone material models," $J$. Biomech., 2017.

[18] T. R. Shultz, J. D. Blaha, T. A. Gruen, and T. L. Norman, "Cortical Bone Viscoelasticity and Fixation Strength of Press-Fit Femoral Stems: A Finite Element Model," J. Biomech. Eng., vol. 128, no. 1, p. 7, 2006.

[19] C. Dopico-González, A. M. New, and M. Browne, "Probabilistic finite element analysis of the uncemented hip replacement-effect of femur characteristics and implant design geometry," J. Biomech., vol. 43, no. 3, pp. 512-520, 2010.

[20] S. Solehuddin, M. I. Z. Ridzwan, M. N. M. Ibrahim, C. J. Tan,' Analysis of Orthopedic Screws for Bone Fracture Fixations with Finite Element Method", Journal of Applied Sciences, 7, 1748-1754, 2007.

[21] D. Y. Ponzio et al., "Intraoperative Proximal Femoral Fracture in Primary Cementless Total Hip Arthroplasty.," J. Arthroplasty, vol. 30, no. 8, pp. 1418-22, 2015. 